\title{
MicroRNA-203-3p inhibits the proliferation, invasion and migration of pancreatic cancer cells by downregulating fibroblast growth factor 2
}

\author{
XI-FENG FU ${ }^{1,2^{*}}$, HAI-CHAO ZHAO ${ }^{1,2^{*}}$, CHUAN-LI YANG ${ }^{1}$, CHANG-ZHOU CHEN ${ }^{2}$, \\ KANG WANG $^{2}$, FEI GAO ${ }^{1}$, YANG-ZHANG TIAN ${ }^{1}$ and HAO-LIANG ZHAO ${ }^{1,2}$ \\ ${ }^{1}$ Department of Biliary and Pancreatic Surgery, Shanxi Bethune Hospital, Shanxi Medical University, Taiyuan, \\ Shanxi 030032; ${ }^{2}$ Third Clinical College, Shanxi Medical University, Taiyuan, Shanxi 030001, P.R. China
}

Received November 19, 2020; Accepted April 23, 2021

DOI: 10.3892/ol.2021.12887

\begin{abstract}
Aberrant expression of fibroblast growth factor 2 (FGF2) is a major cause of poor prognosis in patients with pancreatic cancer. MicroRNA (miRNA/miR) miR-203-3p is a newly identified miRNA that can affect the biological behavior of tumors. The present study investigated the function of miR-203-3p on the regulation of FGF2 expression, and its role in pancreatic cancer cell proliferation, apoptosis, invasion and migration. Reverse transcription-quantitative PCR was used to determine the mRNA expression levels of miR-203-3p and FGF2 in vitro. Cell Counting Kit-8, Annexin V-APC/7-AAD double-staining Apoptosis Detection kit, wound healing and Transwell assays were used to determine the proliferation, apoptosis, migration and invasion of pancreatic cancer cells. The binding of miR-203-3p to FGF2 was assessed by a luciferase reporter assay. The results demonstrated that miR-203-3p expression was downregulated in pancreatic cancer cells. Gainand loss-of-function experiments indicated that miR-203-3p inhibited the proliferation, migration and invasion, and promoted the apoptosis of pancreatic cancer cells in vitro. In addition, it was found that alteration of miR-203-3p abolished the promoting effects of FGF2 on pancreatic cancer cells. The present study demonstrated that FGF2 significantly promoted the proliferation, invasion and migration of pancreatic cancer cells. The mechanism involved the binding of miR-203-3p to the 3'-untranslated region of FGF2 mRNA, resulting in the downregulation of FGF2. In conclusion, miR-203-3p inhibited
\end{abstract}

Correspondence to: Professor Hao-Liang Zhao, Department of Biliary and Pancreatic Surgery, Shanxi Bethune Hospital, Shanxi Medical University, 56 Xinjian South Road, Taiyuan, Shanxi 030032, P.R. China

E-mail: zhaohlxueshu@163.com

*Contributed equally

Key words: microRNA-203-3p, fibroblast growth factor 2, pancreatic cancer, proliferation, migration, invasion
FGF2 expression, regulated the proliferation and inhibited the invasion and migration of pancreatic cancer cells.

\section{Introduction}

Pancreatic cancer poses a major threat to human health, and its incidence is rapidly rising. Cancer data in 2020 revealed that the death toll from pancreatic cancer reached 470,000 worldwide, making it the seventh tumor in terms of cancer-associated deaths; additionally, pancreatic cancer is the tumor with the lowest 5 -year survival rate among all types of tumor (1). Aggressive metastasis is the most important cause of the high mortality rate observed among patients with pancreatic cancer (2). However, the mechanism of metastasis remains unknown. The target molecules that underline metastasis are of great importance in the treatment of pancreatic cancer. Pancreatic ductal adenocarcinoma (PDAC) is the most common type of pancreatic cancer and one of the most challenging malignant tumors to treat (3). The median survival time of patients with PDAC after diagnosis is 2-8 months, and the 5-year overall survival rate is $<7 \%(1,4)$. The poor prognosis in patients with pancreatic cancer is mainly due to the aggressiveness of cancer cells, early metastasis and non-responsiveness to the majority of chemotherapy regimens (5). At present, surgical resection is the only feasible treatment for PDAC. However, $<20 \%$ of tumors are resectable at the time of diagnosis (6). Furthermore, patients who undergo surgery may relapse, and the average survival time of patients undergoing resection is $12-20$ months (7). The majority of patients with pancreatic cancer have early metastatic or locally advanced cancer at the time of diagnosis, and the only effective treatment for these patients is chemoradiation (8). However, pancreatic cancer cells respond poorly to both chemotherapy and radiation $(9,10)$. Although gemcitabine-based chemotherapy, as the standard treatment for advanced pancreatic cancer, can improve prognosis, its effectiveness is limited since cancer cells often become resistant (11). Therefore, further research on pancreatic cancer is urgently needed, and novel diagnostic and therapeutic approaches are required to improve the prognosis of this disease.

Non-coding RNAs (ncRNAs) serve important roles in tumor development. MicroRNAs (miRNAs/miRs) are highly 
conserved single-stranded ncRNA molecules with a length of 18-25 nucleotides. miRNAs can regulate gene expression by base-pairing with 3'-untranslated regions (3'-UTRs), thereby enhancing mRNA degradation or inhibiting post-transcriptional translation (12). To date, $>2,500$ miRNAs have been identified in plants, animals and viruses (13). After miRNAs are produced in the cell nucleus, they are delivered into the cytoplasm by nuclear transporters and then guided into the RNA-induced silencing complex (RISC), where they facilitate target gene mRNA degradation or inhibit translation through complementary pairing with target gene mRNA bases (14). miRNAs serve important roles in tumor development $(15,16)$. For example, previous studies have reported the involvement of various miRNAs (such as miR-21, miR-155 and miR-210) in the development and progression of pancreatic cancer $(17,18)$.

Fibroblast growth factor 2 (FGF2) is the main factor leading to tissue fibrosis, and pancreatic fibrosis plays a key role in the progression of pancreatic cancer (19). Sakai et al (20) found that, when mice were subcutaneously inoculated with BxPC-3 pancreatic cancer cells and simultaneously administered FGF2, the area of interstitial fibrosis increased compared with that of mice administered with BxPC-3 alone. The fibrosis was characterized by increased accumulation of murine collagen, which was associated with an increase in monocyte/macrophage content in tumor tissue, and promoted the progression of pancreatic cancer (20).

miR-203 is located on chromosome 14q32.33. Compared with that in normal tissues, miR-203 exhibits downregulated expression in diverse malignancies, including bladder, non-small cell lung and endometrial cancer (21-23). Numerous studies have demonstrated that miR-203 serves an important role in tumor cell proliferation, migration and invasion $(24,25)$. For example, miR-203 expression is decreased in pancreatic cancer compared with that in normal pancreatic tissue and chronic pancreatitis, suggesting that miR-203 may be associated with specific characteristics of tumors and their behavior (26). Other studies have suggested that FGF2 expression may be affected by miR-203 $(27,28)$. However, the relevant mechanisms of action and signaling pathways in pancreatic cancer remain unknown. The present study established cell models of miR-203 knockdown and overexpression to explore the regulatory effects of miR-203 on FGF2 expression, as well as on the proliferation, invasion and migration of pancreatic cancer cells.

\section{Materials and methods}

Reagents and materials. Pancreatic cancer cell lines [PANC-1 (CVCL_0480), AsPC-1 (CVCL_0152), BxPC-3 (CVCL_0186) and HPAC] were purchased from The Cell Bank of Type Culture Collection of the Chinese Academy of Sciences. Normal human pancreatic epithelial cells [hTERT-HPNE E6/E7 (CRL-4036)] and 293T cells were also purchased from The Cell Bank of Type Culture Collection of the Chinese Academy of Sciences (293T cells were used for transfection and luciferase reporter gene experiments). Dulbecco's modified Eagle's medium (DMEM), fetal bovine serum (FBS) and Opti-MEM were procured from Gibco (Thermo Fisher Scientific, Inc.). Lipofectamine ${ }^{\circledR} 2000$ was obtained from Invitrogen (Thermo Fisher Scientific, Inc.). PrimeScript ${ }^{\mathrm{TM}}$ RT kit and SYBR Green dye were purchased from Takara Bio, Inc. Mimic-miR-203-3p, mimic-negative control (NC; scrambled), inhibitor-miR-203-3p and inhibitor-NC (non-targeting) were obtained from Guangzhou RiboBio Co., Ltd. The pGL3 reporter vector and Dual-Glo Luciferase Activity Assay kit were purchased from Promega Corporation. The Annexin V-FITC/PI Double-Staining Cell Apoptosis Assay kit was purchased from Nanjing KeyGen Biotech Co., Ltd.

Cell culture and transfection. PANC-1, AsPC-1, BxPC-3, HPAC and HPNE cells were cultured in DMEM containing $10 \% \mathrm{FBS}$ and $1 \%$ streptomycin at $37^{\circ} \mathrm{C}$ in an atmosphere of $5 \% \mathrm{CO}_{2}$. The cells were passaged at 1:4 or 1:5 ratio and then used for experiments at the logarithmic growth phase. The cells were digested with $0.25 \%$ trypsin, and the digested cells were counted using a hemocytometer and diluted with culture medium to a concentration of $5 \times 10^{4}$ cells $/ \mathrm{ml}$. Subsequently, $100 \mu \mathrm{l}$ cell suspension was added into each well of a 96-well culture plate, and incubated at $37^{\circ} \mathrm{C}$ in an atmosphere of $5 \% \mathrm{CO}_{2}$ for $24 \mathrm{~h}$. According to the experimental results, AsPC-1 cells were selected as the experimental cell line. For cell transfection, $0.25 \mu \mathrm{g}$ miRNA (mimic-miR-203, miR-NC, inhibitor-NC, inhibitor-miR-203) was diluted with $25 \mu 1$ serum-free Opti-MEM, mixed gently and incubated at room temperature for 5 min. Lipofectamine ${ }^{\circledR} 2000(0.5 \mu \mathrm{l})$ was diluted with $25 \mu \mathrm{l}$ serum-free Opti-MEM, mixed gently and incubated at room temperature for $5 \mathrm{~min}$. Both components were subsequently combined, mixed gently and incubated for $20 \mathrm{~min}$ at room temperature. The miRNA-Lipofectamine 2000 mixture was then added to the wells, which contained $50 \mu \mathrm{l}$ Opti-MEM, and gently mixed. The sequences of the miRs transfected into the cells are as follows: Mimic-miR-203, 5'-GUGAAAUGUUUA GGACCACUAG-3'; miR-NC, 5'-UUCUCCGAACGUGUC ACGUTT-3'; inhibitor-NC, 5'-CAGUACUUUUGUGUAGUA CAA-3'; and inhibitor-miR-203, 5'-CUAGUGGUCCUAAAC AUUUCAC-3'. After 4-6 h of incubation at $37^{\circ} \mathrm{C}$, the medium containing the siRNA-Lipofectamine 2000 mixture was carefully aspirated and replaced with DMEM with 5\% FBS and no antibiotics. The culture plate was incubated at $37^{\circ} \mathrm{C}$ for $24 \mathrm{~h}$ before detection of cell proliferation, apoptosis, invasion and migration. In addition, transfection of FGF2 recombinant plasmid (Guangzhou RiboBio Co., Ltd.; $25 \mathrm{nM} / \mathrm{ml}$; control plasmid was an empty vector) was performed. The specific method was the same as aforementioned for the transfection of mimic/inhibitor-miR-203. Regarding the cell culture with gemcitabine, $0.04 \mu \mathrm{g} / \mathrm{ml}$ gemcitabine (LY 188011; MedChemExpress) was added as previously described (29).

Detection of cell proliferation by the Cell Counting Kit (CCK)-8 assay. After $24 \mathrm{~h}$ of transfection as aforementioned, $10 \mu$ l CCK-8 reagent (Wuhan Boster Biological Technology Co., Ltd.) was added to each well. The culture plate was incubated for $3 \mathrm{~h}$ at $37^{\circ} \mathrm{C}$ and mixed gently on a shaker for $10 \mathrm{~min}$. A microplate reader was used to determine the optical density (OD) value of each well at a wavelength of $450 \mathrm{~nm}$. The inhibition rate $=(\mathrm{NC}$ group OD values - experimental group OD values)/NC group OD values x 100 .

Detection of apoptosis by Annexin $V$-APC/7-AAD double-staining. AsPC-1 cells were washed twice with 
PBS, digested with $0.25 \%$ trypsin for $5 \mathrm{~min}$ and centrifuged at $1,000 \mathrm{x}$ g for $5 \mathrm{~min}$ at $4^{\circ} \mathrm{C}$ to collect $5 \times 10^{5}$ cells, which were then resuspended in $500 \mu 1$ binding buffer (Wuhan Boster Biological Technology Co., Ltd.). Subsequently, $5 \mu 1$ Annexin V-APC was added to the cell suspension, followed by mixing, and then $5 \mu 1$ 7-AAD was added, followed by mixing. The samples were incubated at room temperature in the dark for 5-15 min, and apoptosis was detected using a BD LSRII flow cytometer (BD Biosciences). The detection data were analyzed and graphed using CellQuest Pro (BD Biosciences).

Detection of migration and invasion by wound healing and Transwell assays. For wound healing assays, cells at the logarithmic growth phase were cultured to reached $100 \%$ confluence in 6-well plates. The next day, the AsPC-1 cell layer was scratched with a $10-\mu l$ micropipette tip in the center of the well (denoted as $0 \mathrm{~h}$ ). DMEM with $1 \%$ FBS was used to avoid cell proliferation. The AsPC-1 cells were gently rinsed with PBS and incubated in $1 \%$ FBS-containing DMEM. After $24 \mathrm{~h}$ of incubation at $37^{\circ} \mathrm{C}$, the cells were removed from the incubator, photographed using an inverted light microscope (magnification, x200; Leica Microsystems, Inc.), and the cell migration distance was measured. The width of the wound healing site was quantified and compared with baseline values. All experiments were repeated independently in triplicate.

Cancer cell invasion was tested using Transwell assays. Cells were removed from serum-containing medium and serum-starved for $24 \mathrm{~h}$ using serum-free medium. Matrigel (BD Biosciences) was thawed at $4^{\circ} \mathrm{C}$ overnight, and $2 \times 10^{4}$ cells from each group in $200 \mu \mathrm{l}$ serum-free medium were seeded in the upper chamber (pore size, $8.0 \mu \mathrm{m}$; Corning, Inc.) precoated with $90 \mu \mathrm{l}$ Matrigel at $37^{\circ} \mathrm{C}$ for $8 \mathrm{~h}$. Subsequently, $600 \mu \mathrm{l}$ RPMI-1640 medium (Wuhan Boster Biological Technology Co., Ltd.) containing $10 \%$ FBS was added to the lower chamber. After $24 \mathrm{~h}$ of incubation at $37^{\circ} \mathrm{C}$, the upper chambers were fixed with $4 \%$ polymethanol (Wuhan Boster Biological Technology Co., Ltd.) for $30 \mathrm{~min}$ at room temperature, and then stained with $0.1 \%$ crystal violet for $30 \mathrm{~min}$ at room temperature. The cells that migrated through the membrane and invaded the underside of the upper chamber were photographed using an inverted light microscope (magnification, x200; Leica Microsystems, Inc.). Five random fields were selected to calculate the number of migrating or invading cells.

Reverse transcription-quantitative PCR (RT-qPCR). Total RNA was extracted from cells using TRIzol ${ }^{\circledR}$ reagent (Invitrogen; Thermo Fisher Scientific, Inc.) and reverse transcribed into cDNA according to the manufacturer's protocol using PrimeScript ${ }^{\mathrm{TM}}$ RT kit (Thermo Fisher Scientific, Inc.). RT-qPCR was performed on a CFX96 System (Bio-Rad Laboratories, Inc.). The reactions consisted of 5.0 $\mu 12$ X SYBR Green Master Mix, $0.5 \mu \mathrm{l}$ each forward and reverse primers $(2.5 \mu \mathrm{M}), 1 \mu \mathrm{l}$ cDNA and RNase-free double-distilled $\mathrm{H}_{2} \mathrm{O}$. The thermocycling conditions were as follows: $95^{\circ} \mathrm{C}$ for $5 \mathrm{~min}$, followed by 40 cycles of $95^{\circ} \mathrm{C}$ for $15 \mathrm{sec}$ and $60^{\circ} \mathrm{C}$ for $1 \mathrm{~min}$. The relative expression of target mRNA and miRNA was quantified using the $2^{-\Delta \Delta \mathrm{Cq}}$ method (30). GAPDH was used as the reference gene to normalize mRNA and U6 was used as the reference gene to normalize miRNA expression. The primer sequences are shown in Table SI.
Western blot analysis. Cells were washed with PBS to extract the total protein using RIPA Lysis and Extraction Buffer (Wuhan Boster Biological Technology Co., Ltd.). The concentration of the extracted protein was measured with a BCA protein quantification kit (Wuhan Boster Biological Technology Co., Ltd.) to prepare samples for electrophoresis. Subsequently, samples were mixed with a loading buffer (Wuhan Boster Biological Technology Co., Ltd.) at a ratio of 5:1 and subjected to heat at $100^{\circ} \mathrm{C}$ for $10 \mathrm{~min}$. A total of $50 \mu \mathrm{g}$ protein/lane from each sample was separated by $10 \%$ SDS-PAGE and then transferred to a PVDF membrane. The membrane was blocked with $5 \%$ skimmed milk powder in TBS-0.5\% Tween (TBST) at room temperature for $2 \mathrm{~h}$ and then incubated with the following anti-human primary antibodies at $4^{\circ} \mathrm{C}$ for 12 h: FGF2 (cat. no. BM4959; 1:1,000), FGF receptor 3 (FGFR3; cat. no. BM5016; 1:500), FGFR2 (cat. no. BM4991; 1:1,000) and GAPDH (cat. no. BM3896; 1:10,000) (all Wuhan Boster Biological Technology Co., Ltd.). Subsequently, a secondary antibody (HRP-conjugated affiniPure goat anti-rabbit IgG; cat. no. BA1055, 1:5,000; Wuhan Boster Biological Technology Co., Ltd.) was incubated with the membrane at $37^{\circ} \mathrm{C}$ for $2 \mathrm{~h}$, and an $\mathrm{ECL}^{\mathrm{TM}}$ reagent (Wuhan Boster Biological Technology Co., Ltd.) was used for visualizing the protein bands after washing with TBST 3 times for 19 min each. GADPH was used as a loading control for normalization. The results were analyzed using ImageJ software (v1.8.0; National Institutes of Health).

Bioinformatics analysis. TargetScan (http://www.targetscan. org/vert_72/) is a web server that predicts biological targets of miRNAs by searching for the presence of sites that match the seed region of each miRNA. FGF2 and miR-203-3p were inserted to analyze whether they could bind to each other and to identify the binding sequence online.

Dual-luciferase reporter assay. The wild-type (WT) FGF2 fragments containing the putative miR-203-3p binding site were prepared and cloned into the pGL3 reporter vectors (Promega Corporation) to yield the FGF2-WT reporter constructs. The mutated (Mut) versions of the fragments were additionally prepared according to the manufacturer's protocol with the GeneTailor ${ }^{\mathrm{TM}}$ Site-Directed Mutagenesis System (Invitrogen; Thermo Fisher Scientific, Inc.), after which FGF2-Mut reporter constructs were generated as aforementioned. After growing until 70-80\% confluence, cotransfection of WT or Mut reporter vectors with miR-203-3p mimic or NC mimic was implemented using Lipofectamine 2000, as aforementioned. After $36 \mathrm{~h}$ of incubation at $37^{\circ} \mathrm{C}$, firefly luciferase activity was detected according to the manufacturer's instructions of the Dual-Glo Luciferase Activity Assay kit and compared with Renilla luciferase activity. All experiments were repeated independently in triplicate.

Statistical analysis. The experimental results are presented as the mean \pm SD and all experiments were repeated $\geq 3$ times. SPSS 26.0 Software (IBM Corp.) was used for statistical analysis. According to whether the data conformed to the normal distribution, The data of CCK-8, apoptosis, migration, invasion and RT-qPCR assays were analyzed according to data types and comparison methods. Kruskal-Wallis test 

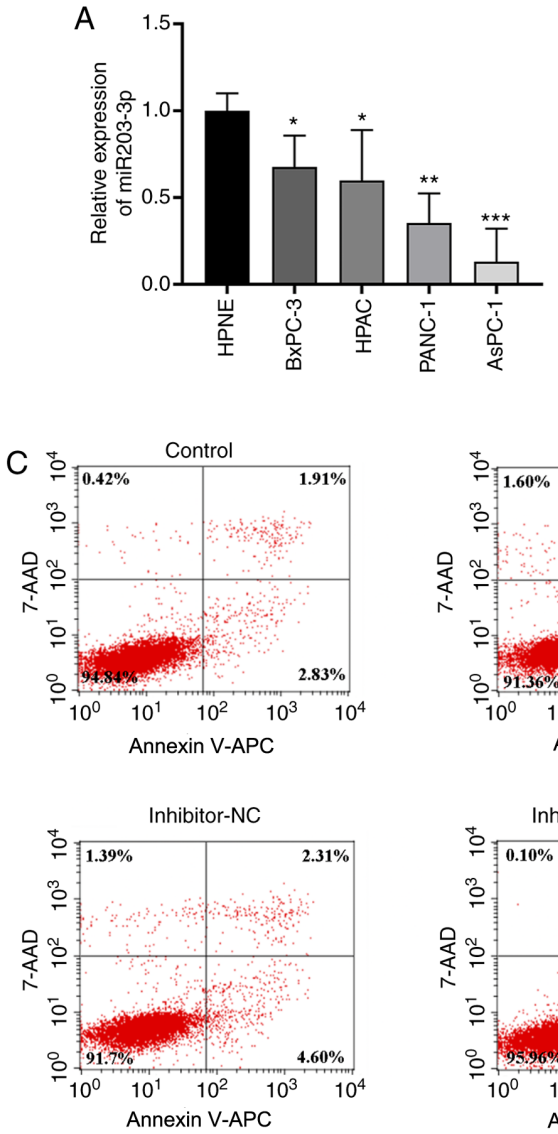
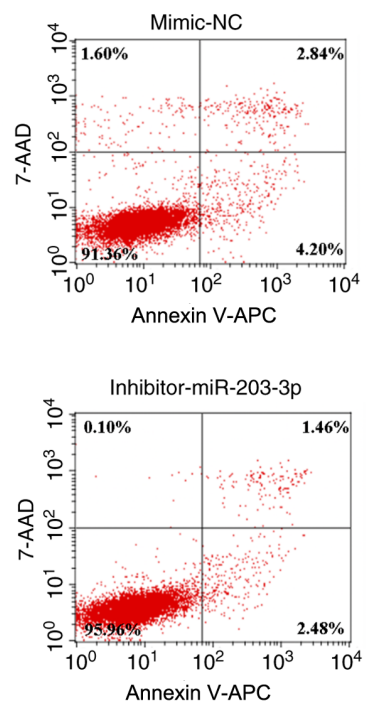
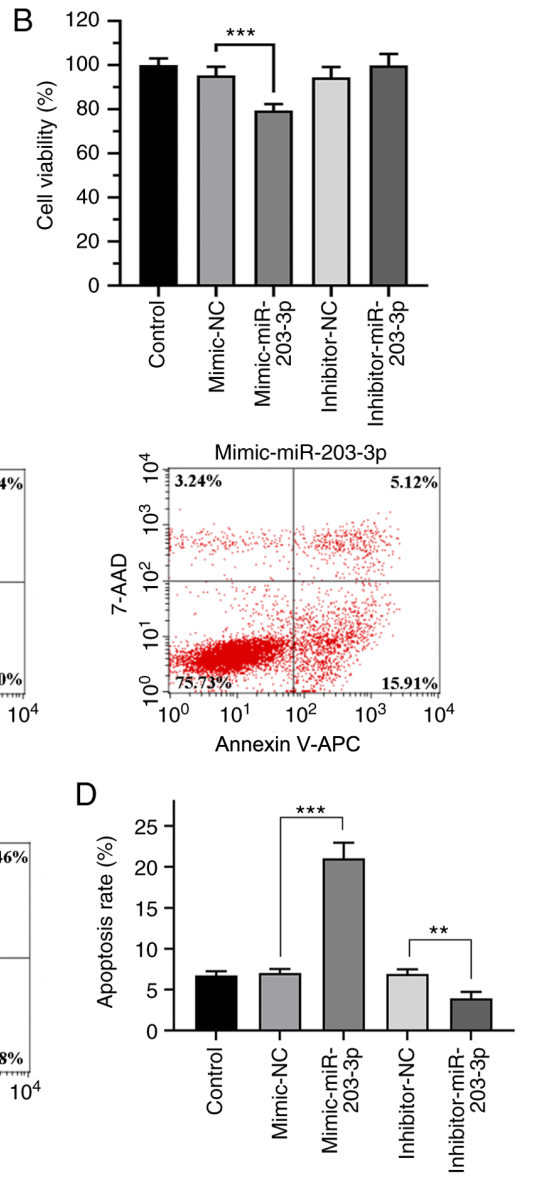

Figure 1. miR-203-3p overexpression decrease the viability and increases the apoptosis of pancreatic cancer cells. (A) Relative miR-203-3p expression was detected in pancreatic cancer cell lines by reverse transcription-quantitative PCR. ${ }^{*} \mathrm{P}<0.05,{ }^{* *} \mathrm{P}<0.01$ and ${ }^{* * *} \mathrm{P}<0.001$ vs. HPNE . (B) miR-203a-3p overexpression inhibited the viability of the pancreatic cancer AsPC-1 cell line, as detected by Cell Counting Kit-8 assay. (C) miR-203a-3p overexpression increased AsPC-1 cell apoptosis, as detected by Annexin V-APC/7-AAD double staining. (D) Apoptosis rate of AsPC-1 cells treated with mimic-NC, mimic-miR-203-3p, inhibitor-NC or inhibitor-miR-203-3p. Control represents AsPC-1 cells without transfection. All data are presented as the mean \pm SD of three experiments. ${ }^{* * *} \mathrm{P}<0.01 ;{ }^{* * *} \mathrm{P}<0.001 . \mathrm{miR}$, microRNA; NC, negative control.

was used for comparison between two groups (e.g. mimic-NC vs. mimic-miR, inhibitor-NC vs. inhibitor-miR, AsPC1-NC vs. AsPC1-FGF2), and the Bonferroni method was used for multiple comparisons. Statistical comparison of mean values in two groups were compared by unpaired Student's t-test. Multiple groups were compared by one-way ANOVA with Dunnett's post hoc test. All graphs were produced using GraphPad Prism (version 8.0; GraphPad Software, Inc.) and ImageJ (v1.8.0; National Institutes of Health). $\mathrm{P}<0.05$ was considered to indicate a statistically significant difference.

\section{Results}

miR-203-3p expression in different cell lines. RT-qPCR was used to quantify miR-203-3p expression in the HPNE, BxPC-3, HPAC, PANC-1 and AsPC-1 cell lines, and it was found that miR-203-3p expression was significantly lower in pancreatic cancer cells than that in HPNE cells (Fig. 1A). Furthermore, miR-203-3p expression was the lowest in AsPC-1 cells (Fig. 1A), indicating that the AsPC-1 cell line was suitable for further experiments.

miR-203-3p inhibits the proliferation of AsPC-1 cells. To investigate the role of miR-203-3p in pancreatic cancer cells,
inhibitor-miR-203-3p was used to knockdown miR-203-3p, which targeted the junction sites of miR-203-3p. Overexpression of miR-203-3p was achieved using mimic-miR-203-3p for transfection into AsPC-1 cells. The results of the CCK-8 assay revealed that miR-203-3p overexpression significantly inhibited the proliferation of AsPC-1 cells (Fig. 1B). As shown in Table SII, compared with that of the mimic-NC group, the inhibition rate of the mimic-miR-203-3p group was increased. However, compared with that of the inhibitor-NC group, the inhibition rate of the inhibitor-miR-203-3p group did not markedly change (Fig. 1B and Table SII).

miR-203-3p increases the apoptosis of pancreatic cancer cells. The AsPC-1 cell apoptosis rate was significantly different among all groups. Compared with that of the mimic-NC group, the apoptosis rate of the mimic-miR-203-3p group was significantly increased, while compared with that of the inhibitor-NC group, the apoptosis rate of the inhibitor-miR-203-3p group was significantly decreased (Fig. 1C and D, and Table SIII).

miR-203-3p decreases cell migration and invasion, and increases the sensitivity of pancreatic cancer cells to gemcitabine. The present study examined the ability of cells 
A

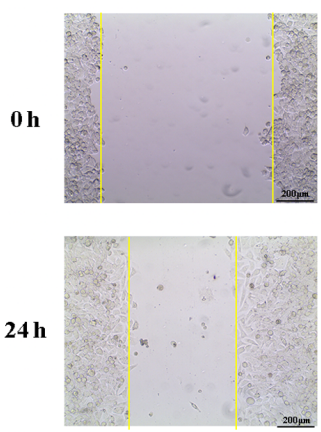

B
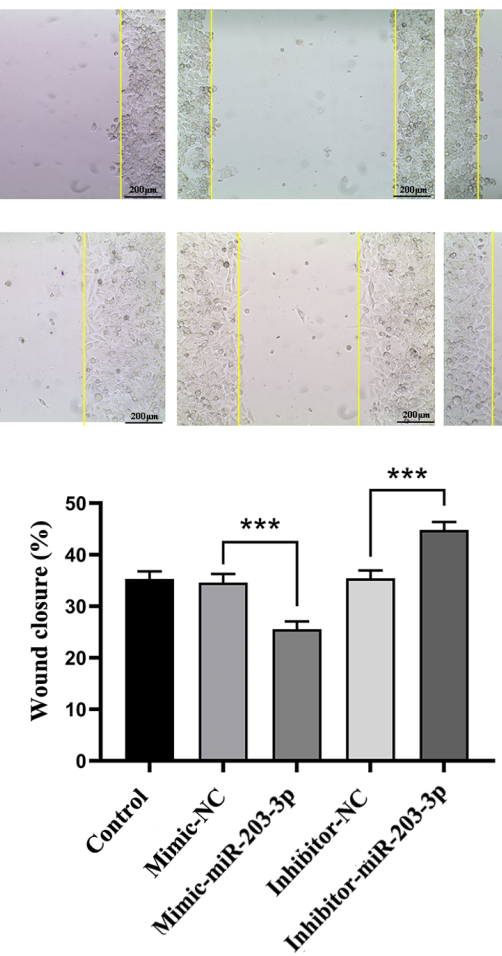

Mimic-miR-203-3p
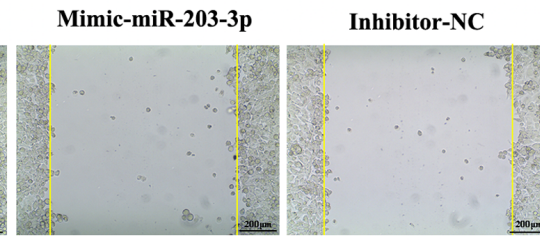

Inhibitor-miR-203-3p
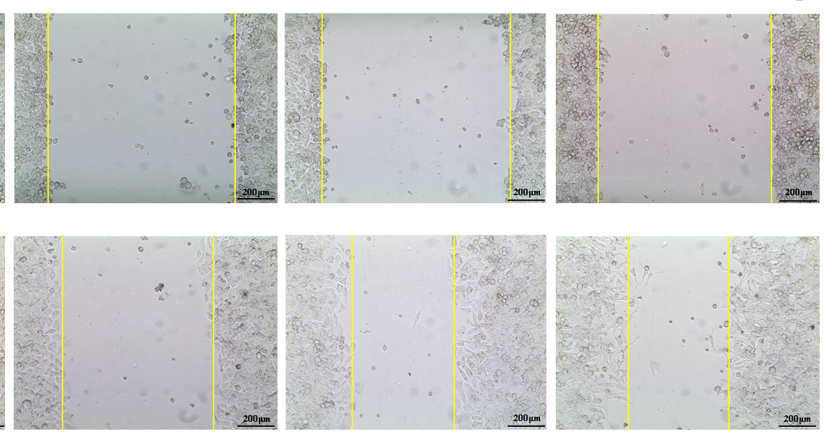

C

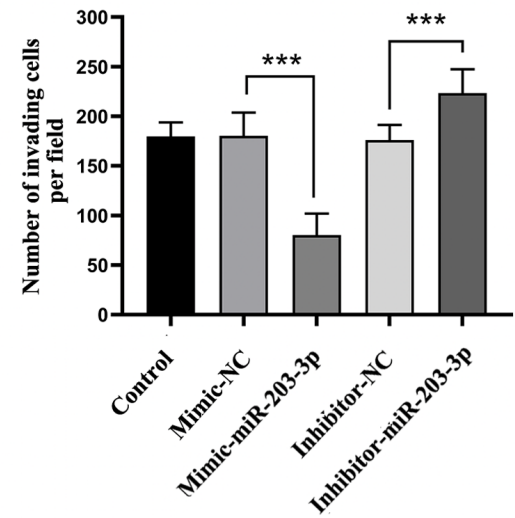

D

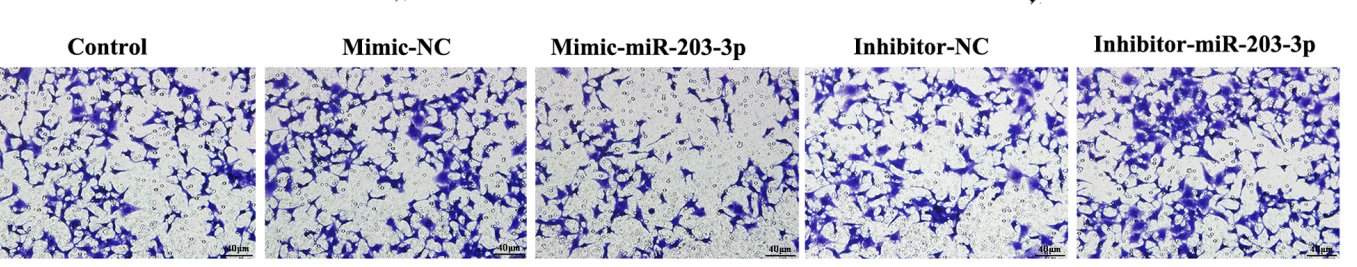

E

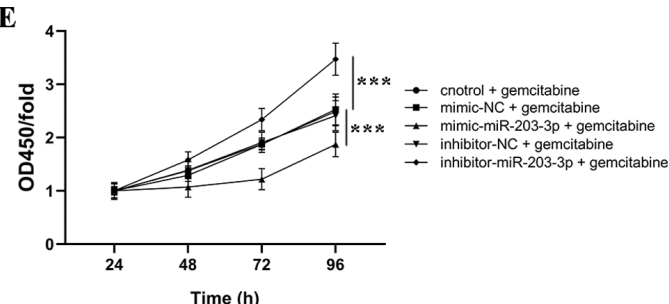

Figure 2. miR-203-3p overexpression decreases the migration and invasion of pancreatic cancer cells. (A) Effects of miR-203-3p overexpression or knockdown expression on cell migration were detected by wound healing assay (scale bar, $200 \mu \mathrm{m}$ ). (B) Wound closure percentage of AsPC-1 cells treated with mimic-NC, mimic-miR-203-3p, inhibitor-NC or inhibitor-miR-203-3p. (C) Number of invading AsPC-1 cells treated with mimic-NC, mimic-miR-203-3p, inhibitor-NC or inhibitor-miR-203-3p group. (D) Effects of miR-203-3p overexpression or knockdown on cell invasion were detected by Transwell assay (scale bar, $40 \mu \mathrm{m}$ ). (E) Proliferation curve of AsPC-1 cells treated with mimic-NC, mimic-miR-203-3p, inhibitor-NC or inhibitor-miR-203-3p group. Control represents AsPC-1 cells without transfection. All data are presented as the mean $\pm \mathrm{SD}$ of three experiments. ${ }^{* * *} \mathrm{P}<0.001$. OD, optical density; miR, microRNA; NC, negative control.

transfected with mimic- or inhibitor-miR-203-3p to invade and migrate. Compared with that of the mimic-NC group, the mimic-miR-203-3p group exhibited significantly decreased migration and invasion at $24 \mathrm{~h}$, while knockdown of miR-203-3p expression significantly promoted cell migration and invasion (Fig. 2A-D). Compared with the gemcitabine group and the mimic-NC + gemcitabine group, the proliferation curve of the AsPC-1 cells in the mimic-miR-203-3p + gemcitabine group was significantly decreased; however, compared with the gemcitabine group and the inhibitor-NC + gemcitabine group, the inhibitor-miR-203-3p + gemcitabine group exhibited higher proliferation rates at $96 \mathrm{~h}$ (Fig. 2E). Overall, the current findings suggested that miR-203-3p restrained the progression of pancreatic cancer and increased the sensitivity of pancreatic cancer cells to gemcitabine in vitro.

miR-203-3p inhibits FGF2 expression in pancreatic cancer cells. miR-203-3p expression was significantly decreased by inhibitor-miR-203-3p, whereas it was significantly enhanced by mimic-miR-203-3p (Fig. 3A). Additionally, it was found that miR-203-3p overexpression in AsPC-1 cells significantly decreased FGF2 mRNA expression, while miRNA-203-knockdown in AsPC-1 cells significantly increased FGF2 mRNA expression, as determined by RT-qPCR (Fig. 3A). Furthermore, the protein expression levels of FGFR2 and FGFR3 were detected by western blotting, revealing that miR-203-3p 

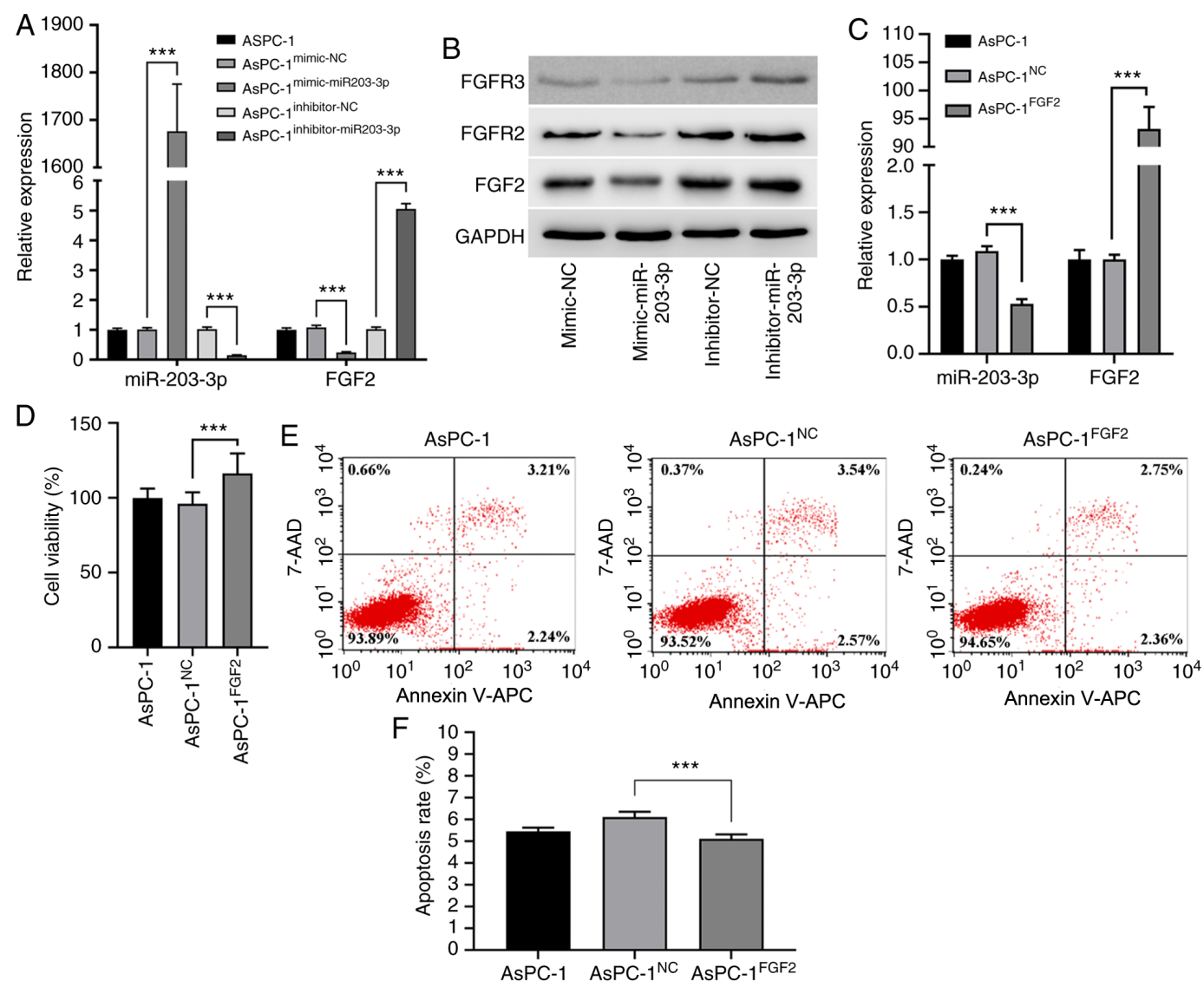

Figure 3. Overexpression of miR-203-3p leads to inhibition of FGF2 expression in pancreatic cancer cells. (A) RT-qPCR revealed the relative expression levels of miR-203-3p and FGF2 in pancreatic cancer AsPC-1 cells with either overexpression or knockdown of miR-203-3p. (B) Protein expression levels of FGF2 and FGFRs were detected by western botting. (C) Relative expression levels of miR-203-3p and FGF2 in pancreatic cancer cells after FGF2 overexpression were detected by RT-qPCR. (D) Cell viability was determined by Cell Counting Kit-8 assay. (E) FGF2 suppressed the apoptosis of pancreatic cancer cells, as detected by Annexin V-APC/7-AAD double staining. (F) Bar graphs representing the quantification of the apoptosis rate. All data are presented as the mean \pm SD of three experiments. ${ }^{* * *} \mathrm{P}<0.001$. miR, microRNA; FGF2, fibroblast growth factor 2; FGFR, FGF receptor; RT-qPCR, reverse transcription-quantitative PCR; NC, negative control.

overexpression decreased FGFR2 and FGFR3 expression, while miR-203-3p-knockdown increased FGFR2 and FGFR3 expression (Fig. 3B). These results indicated that upregulated miR-203-3p expression elicited inhibitory effects on FGF2 expression.

To verify the role of FGF2 in AsPC-1 cells, the control plasmid and the FGF2 overexpression plasmid were simultaneously transfected, and RT-qPCR was performed. It was found that FGF2 mRNA expression in the AsPC-1-FGF2 group increased significantly compared with that in the AsPC-1-NC group (Fig. 3C). In addition, miR-203 was significantly decreased after FGF2 overexpression (Fig. 3C).

FGF2 significantly affects the proliferation of AsPC-1 cells. FGF2 overexpression in pancreatic cancer cells significantly affected cell proliferation. Compared with that of the AsPC-1 and AsPC-1-NC groups, the cell viability of the AsPC-1-FGF2 group was significantly increased, indicating that FGF2 expression promoted the proliferation of AsPC-1 cells (Fig. 3D and Table SIV).

FGF2 affects AsPC-1 cell apoptosis. The flow cytometry results indicated that the apoptosis rate was significantly decreased in the AsPC-1-FGF2 group compared with that in the AsPC-1-NC group, indicating that the change in FGF2 expression significantly affected apoptosis (Fig. 3E and F).

Overexpression of FGF2 increases cell migration and invasion, and the resistance of pancreatic cancer cell lines to gemcitabine. Compared with the AsPC-1 ${ }^{\mathrm{NC}}$ group, the wound closure ratio was significantly increased in the AsPC-1 ${ }^{\text {FGF2 }}$ group (Fig. 4A and B). Similarly, compared with the AsPC- $1^{\mathrm{NC}}$ group, the invading cell number was significantly increased in the AsPC-1 ${ }^{\mathrm{FGF} 2}$ group (Fig. 4C and D). These results indicated that $\mathrm{FGF} 2$ promoted the migratory and invasive abilities of the AsPC-1 pancreatic cancer cell line. Compared with the gemcitabine group and the $\mathrm{NC}+$ gemcitabine group, the AsPC-1 cells of the FGF2 + gemcitabine group exhibited significantly higher proliferation efficiency (Fig. 4E).

miR-203-3p directly interacts with the 3'-UTR region of FGF2 in pancreatic cancer cells. According to bioinformatics analysis using TargetScan, a binding site to the 3'-UTR of FGF2 was predicted in miR-203-3p (Fig. 5A). A dual-luciferase reporter system was used to verify whether miR-203-3p interacted with the binding site of the 3'-UTR of FGF2. A dual-luciferase reporter gene vector for the target gene site region (including WT and Mut) was successfully 
A

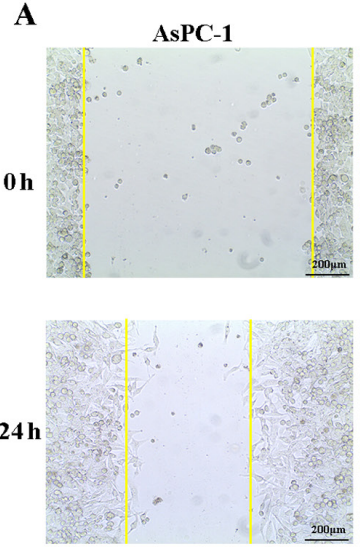

C
AsPC-1 $^{\text {NC }}$
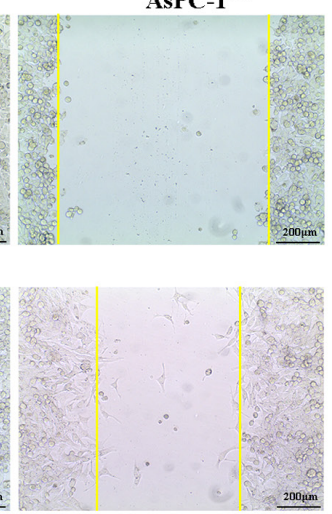

AsPC-1 $^{\text {NC }}$
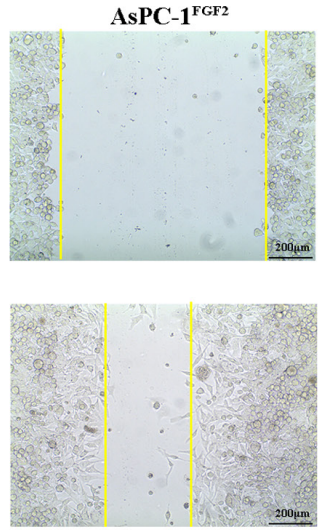

AsPC-1 ${ }^{\text {FGF2 }}$

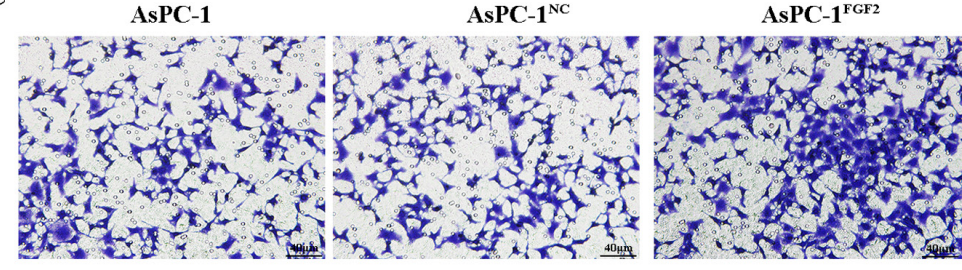

$\mathbf{E}$

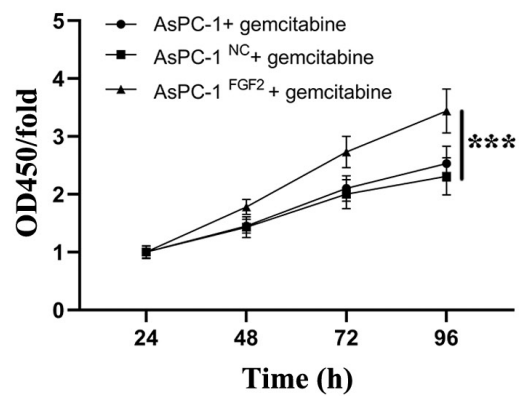

B

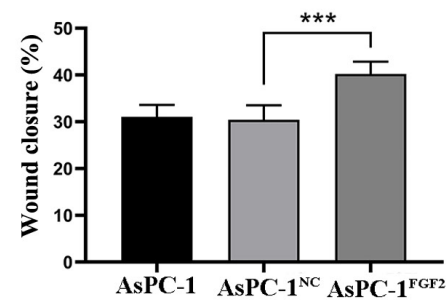

D

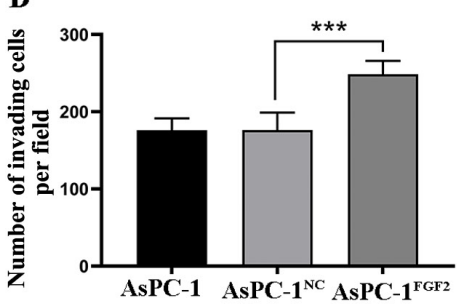

Figure 4. Overexpression of FGF2 increases the migration and invasion of pancreatic cancer cells. (A) Wound healing assays were used to detect the migration of AsPC-1, AsPC-1-NC and AsPC-1-FGF2 cell lines (scale bar, $200 \mu \mathrm{m}$ ). (B) Wound closure percentage of AsPC-1, AsPC-1-NC and AsPC-1-FGF2 cells. (C) Transwell assays were used to detect the invasion of AsPC-1, AsPC-1-NC and AsPC-1-FGF2 cells (scale bar, $40 \mu \mathrm{m}$ ). (D) Number of invading AsPC-1, AsPC-1-NC and AsPC-1-FGF2 cells. (E) Proliferation curve of AsPC-1, AsPC-1-NC and AsPC-1-FGF2 group. ${ }^{* * *}$ P<0.001. FGF2, fibroblast growth factor 2; $\mathrm{NC}$, negative control; OD, optical density.

constructed, and was co-transfected with mimic-miR-203a-3p and mimic-NC. The luciferase activity in transfected cells was determined. The results revealed that luciferase activity in AsPC-1 cells co-transfected with mimic-miR-203-3p and WT vectors was significantly decreased compared with that of cells transfected with MUT vectors (Fig. 5B). These results indicated that miR-203-3p interacted directly with the 3'-UTR of FGF2. This mechanism of direct binding indicated that miR-203-3p directly regulated its target gene FGF2.

\section{Discussion}

Since no significant progress has been made lately in the treatment of pancreatic cancer, research has focused on the tumor cells per se, as well as on the tumor immune microenvironment of pancreatic cancer (31). Previous studies have found that overexpression of FGF2 and its receptor in pancreatic cancer cells in the tumor microenvironment promotes the progression of the tumor itself, and is associated with a poor prognosis (32-34). Clinically resected pancreatic cancer specimens, particularly PDAC specimens, have a hard consistency, and large quantities of collagen can be observed by hematoxylin and eosin staining under an optical microscope (35). Kostas et al (36) described the anti-apoptotic effects of FGF1 and FGF2 in cells, which were independent of FGFR activation and downstream signaling. Shirakihara et al (37) found that FGF2 can promote epithelial-mesenchymal transition (EMT) in injured tissues. Our previous study has revealed that the heparanase/syndecan-1 axis can upregulate the FGF2 level and increase the expression levels of downstream Palladin by activating the PI3K/Akt signaling pathway, thereby leading to the activation of EMT (38). It has been also reported that EMT promotes the migration and invasion of pancreatic cancer cells (38). Therefore, it is necessary to identify a method that can interfere with the expression of target proteins.

miRNA is a type of post-transcriptional regulatory factor that does not directly act on genes, but downregulates the expression levels of target genes by acting on the mRNA transcribed from genes (39). The present study detected miR-203-3p expression in pancreatic cancer cell lines (PANC-1, AsPC-1, BxPC-3 and HPAC) and in normal pancreatic cells (HPNE). Previous studies have investigated the role of miR-203 in 

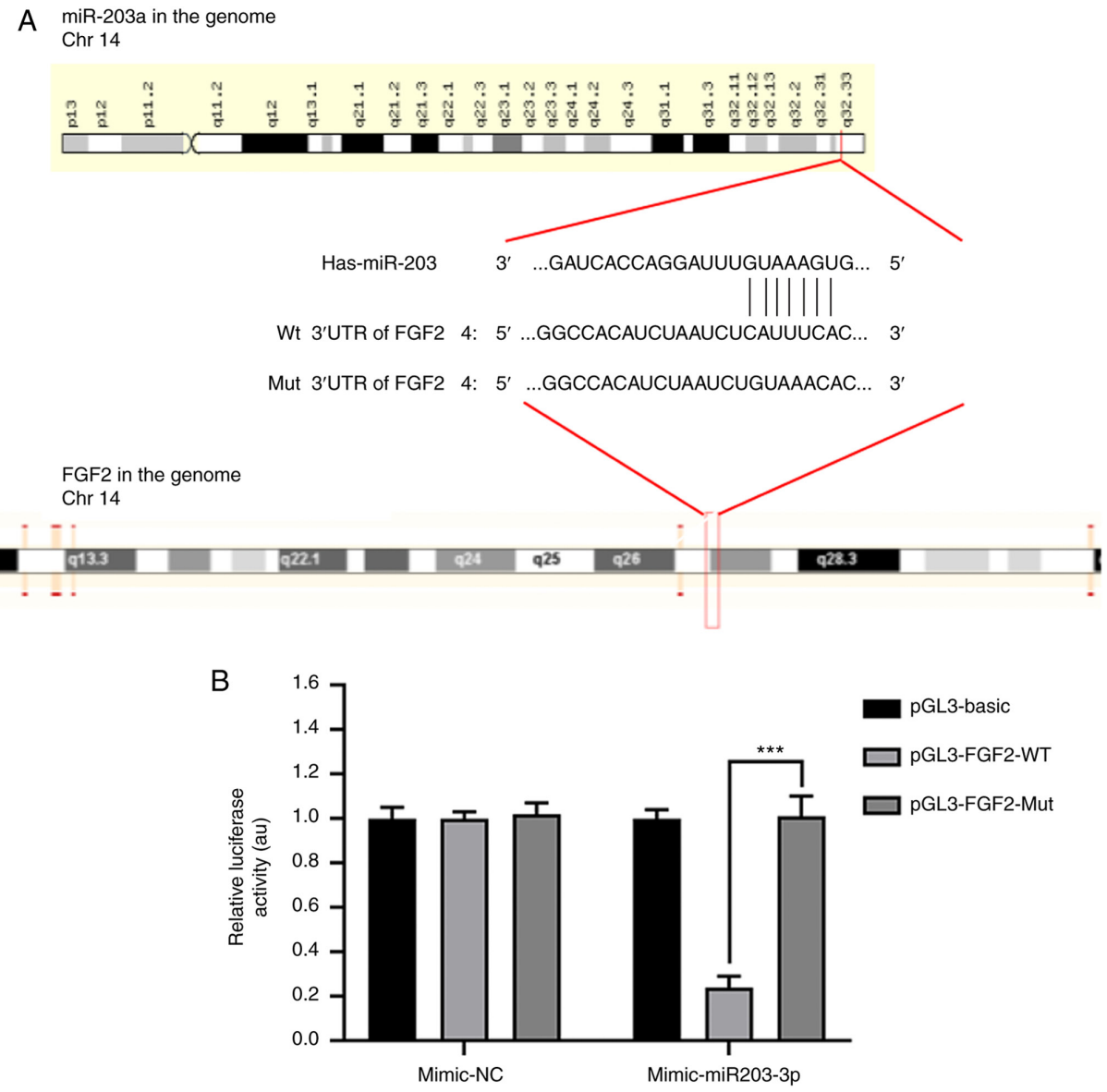

Figure 5. miR-203-3p directly targets FGF2 in pancreatic cancer cells. (A) Sequence alignment of miR-203-3p and 3'-UTR of FGF2. (B) Results of the luciferase assay performed after co-transfecting cells with miR-203-3p mimic and a luciferase reporter vector containing the WT or Mut 3'-UTR of FGF2. All data are presented as the mean $\pm \mathrm{SD}$ of three experiments. ${ }^{* * * *} \mathrm{P}<0.001$. miR, microRNA; FGF2, fibroblast growth factor 2; UTR, untranslated region; WT, wild-type; Mut, mutated.

tumors $(24,25,39)$. The present study found that miR-203-3p expression was significantly lower in pancreatic cancer cell lines compared with in normal pancreatic cells, which is consistent with the results of Du et al (24). Lin et al (25) reported that miR-203-3p expression is increased in pancreatic cancer tissues, but the miRNA detection of tissues may be affected by the presence of lymphocytes, fibroblasts, other interstitial cells and non-cellular components, resulting in inaccurate results (40). Using in vitro experiments, the present study indicated that miR-203-3p expression affected the proliferation, apoptosis, invasion and migration of pancreatic cancer cells. Overexpression of miR-203-3p significantly inhibited the proliferation, invasion and migration of AsPC-1 cells, and promoted their apoptosis. According to our preliminary experiments (38) and the TargetScan sequence prediction results, subsequent FGF2 detection was performed.

Our group has previously demonstrated that FGF2 overexpression can contribute to the resistance of pancreatic cancer cells to chemotherapy $(29,35)$. Patients with pancreatic cancer and high FGF2 expression are not responsive to postoperative chemotherapy with gemcitabine, and the overall prognosis is poor (41). The present study revealed that increased miR-203 expression inhibited the transcription of FGF2 mRNA in AsPC-1 cells, thereby inhibiting the progression of pancreatic cancer cells. In recent years, the association between cytokines and tumorigenesis has gained increasing attention. Cytokine-receptor interactions activate various signaling pathways in cells and serve important roles in regulating cell proliferation, apoptosis and angiogenesis (42). FGF2, a growth-promoting factor, is highly expressed in breast, gastric and thyroid cancer, as well as in other normal tissues, and aberrant FGF2/FGFR1 signaling may promote tumor development (43). miRNAs regulate gene expression after transcription. Therefore, miRNAs are key regulators of gene expression and promising candidates for biomarker development. Additionally, miRNAs guide RISC to degrade mRNAs or prevent translation by base-pairing with target gene mRNAs (12). The present study demonstrated that miR-203-3p, combined with the 3'-UTR of FGF2, exerted a direct regulatory effect. The current results are consistent with those of previous studies $(28,44)$, and may explain why miR-203 affected the proliferation, invasion and migration of pancreatic cancer cells. However, it was also revealed that downregulation of miR-203 inhibited AsPC-1 cell apoptosis, while overexpression of FGF2 promoted the viability of pancreatic cancer cells. Therefore, miR-203 may also affect the viability of AsPC-1 cells through other signaling pathways, and further studies are required to explore this hypothesis.

$\mathrm{Li}$ et al (45) has found that FGF2 prevents cancer cells from endoplasmic reticulum (ER) stress-mediated apoptosis 
via enhancing proteasome-mediated non-catalytic region of tyrosine kinase degradation. The present study indicated that overexpression of FGF2 in pancreatic cancer AsPC-1 cells promoted cell viability and inhibited apoptosis. The specific mechanism may be through FGF2 binding and activating the tumor cell surface tyrosine kinase receptor FGFR. Intracellular signaling (including Ras/MAPK, PI3K/Akt and PLC $\gamma / \mathrm{PKC}$ ) leads to the proliferation, migration or differentiation of multiple types of cells and exerts significant anti-apoptotic effects $(46,47)$. In addition to this classic mode of action, Kostas et al (36) revealed that under ER stress conditions, FGF1 and FGF2 can be transported to the cytoplasm and nucleus through endosomal membranes in a receptor-independent manner to exert their anti-apoptotic effects. FGF2 has significantly stronger anti-apoptotic effects than FGF1 (48). Thus, the present study hypothesized that, when tumor cells are deficient in FGF2, the signaling pathways in tumor cells may be altered, the ER stress may be unbalanced, and apoptosis may increase. This theory is consistent with the present results, since downregulating intracellular FGF2 significantly induced apoptosis.

The regulation of miR-203-3p expression in pancreatic cancer should also be further investigated. Li et al (48) has revealed that epidermal growth factor in esophageal squamous cells acts on the upstream regulatory elements of miR-203 through CCAAT-enhancer binding protein $\beta$ LIP to downregulate miR-203-3p expression in the cells. Zhang et al (49) has demonstrated that cisplatin induces the production of E2F transcription factor 1 (E2F1) in esophageal cancer cells, and then acts on the E2F1 promoter to regulate miR-203 expression. The abnormal expression levels of miR-203-3p in pancreatic cancer cells require to be further studied.

The present study has some limitations. At present, gemcitabine has become the main part of pancreatic cancer drug treatment. In the current study, the joint effect of miR-203-3p and gemcitabine was investigated, but the mechanism was not explored in depth. Moreover, whether miR-203-3p can reverse the gemcitabine resistance of pancreatic cancer cell lines should be further investigated. Second, although miR-203-3p downregulated FGF2 expression, whether it changes the sensitivity of pancreatic cancer cell lines to gemcitabine through FGF2 should also be further studied.

In summary, the present study provided evidence that miRNA-203-3p expression was abnormally low in pancreatic cancer cells, and that miRNA-203-3p overexpression inhibited the proliferation, invasion and migration, and increased the apoptosis of pancreatic cancer cells. Furthermore, the present study has strengthened the evidence that miR-203-3p may directly act on FGF2, thus suggesting the value of miR-203 as an important biomarker for pancreatic cancer progression.

\section{Acknowledgements}

Not applicable.

\section{Funding}

The present study was funded by grants from the Shanxi Science and Technology Department (grant nos. 201901D11412 and 201901D1111404) and Shanxi '136' Leading Clinical Key Specialty (grant nos. 2019XY002 and 2019XY012).

\section{Availability of data and materials}

All data generated or analyzed during this study are included in this published article.

\section{Authors' contributions}

XFF and HCZ conceived the project. XFF, HCZ, CLY, CZC and $\mathrm{KW}$ performed most of the experiments. $\mathrm{HCZ}$ wrote the manuscript and collected the data. CLY and CZC collected and analyzed the data. YZT, KW and FG contributed to the interpretation of the data and the critical review of the manuscript. HLZ made contributions to conception and design, acquisition of data and revision and correction of the manuscript. HLZ, YZT and XFF provided the funding for the study. The authenticity of all the raw data was assessed by YZT and HLZ. All authors have read and approved the final manuscript.

\section{Ethics approval and consent to participate}

Not applicable.

\section{Patient consent for publication}

Not applicable.

\section{Competing interests}

The authors declare that they have no competing interests.

\section{References}

1. Siegel RL, Miller KD and Jemal A: Cancer statistics, 2020. CA Cancer J Clin 70: 7-30, 2020

2. Rawla P, Sunkara T and Gaduputi V: Epidemiology of pancreatic cancer: Global trends, etiology and risk factors. World $\mathbf{J}$ Oncol 10: 10-27, 2019.

3. Adamska A, Domenichini A and Falasca M: Pancreatic ductal adenocarcinoma: Current and evolving therapies. Int J Mol Sci 18: 1338, 2017.

4. Gillen S, Schuster T, Meyer Zum Büschenfelde C, Friess H and Kleeff J: Preoperative/neoadjuvant therapy in pancreatic cancer: A systematic review and meta-analysis of response and resection percentages. PLoS Med 7: e1000267, 2010.

5. Maitra A and Hruban RH: Pancreatic cancer. Annu Rev Pathol 3: $157-188,2008$.

6. Li D, Xie K, Wolff R and Abbruzzese JL: Pancreatic cancer. Lancet 363: 1049-1057, 2004

7. Siegel RL, Fedewa SA, Miller KD, Goding-Sauer A, Pinheiro PS, Martinez-Tyson D and Jemal A: Cancer statistics for hispanics/latinos, 2015. CA Cancer J Clin 65: 457-480, 2015.

8. McGuigan A, Kelly P, Turkington RC, Jones C, Coleman HG and McCain RS: Pancreatic cancer: A review of clinical diagnosis, epidemiology, treatment and outcomes. World J Gastroenterol 24: 4846-4861, 2018

9. Garrido-Laguna I and Hidalgo M: Pancreatic cancer: From state-of-the-art treatments to promising novel therapies. Nat Rev Clin Oncol 12: 319-324, 2015.

10. Neoptolemos JP, Stocken DD, Friess H, Bassi C, Dunn JA, Hickey H, Beger H, Fernandez-Cruz L, Dervenis C, Lacaine F, et al: A randomized trial of chemoradiotherapy and chemotherapy after resection of pancreatic cancer. N Engl J Med 350: 1200-1210, 2004

11. Amrutkar M and Gladhaug IP: Pancreatic cancer chemoresistance to gemcitabine. Cancers (Basel) 9: 157, 2017. 
12. He L and Hannon GJ: MicroRNAs: Small RNAs with a big role in gene regulation. Nat Rev Genet 5: 522-531, 2004.

13. Li H, Jiang JD and Peng ZG: MicroRNA-mediated interactions between host and hepatitis C virus. World J Gastroenterol 22: 1487-1496, 2016.

14. Macfarlane LA and Murphy PR: MicroRNA: Biogenesis, function and role in cancer. Curr Genomics 11: 537-561, 2010.

15. Chen X, Cheng JY and Yin J: Predicting microRNA-disease associations using bipartite local models and hubness-aware regression. RNA Biol 15: 1192-1205, 2018.

16. Hatziapostolou M, Polytarchou C and Iliopoulos D: miRNAs link metabolic reprogramming to oncogenesis. Trends Endocrinol Metab 24: 361-373, 2013

17. Dillhoff M, Liu J, Frankel W, Croce C and Bloomston M: MicroRNA-21 is overexpressed in pancreatic cancer and a potential predictor of survival. J Gastrointest Surg 12: 2171-2176, 2008.

18. Szafranska AE, Davison TS, John J, Cannon T, Sipos B, Maghnouj A, Labourier E and Hahn SA: MicroRNA expression alterations are linked to tumorigenesis and non-neoplastic processes in pancreatic ductal adenocarcinoma. Oncogene 26: 4442-4452, 2007.

19. Marzoq AJ, Giese N, Hoheisel JD and Alhamdani MSS: Proteome variations in pancreatic stellate cells upon stimulation with proinflammatory factors. J Biol Chem 288: 32517-32527, 2013.

20. Sakai S, Iwata C, Tanaka HY, Cabral H, Morishita Y, Miyazono K and Kano MR: Increased fibrosis and impaired intratumoral accumulation of macromolecules in a murine model of pancreatic cancer co-administered with FGF-2. J Control Release 230 109-115, 2016.

21. Shen J, Zhang J, Xiao M, Yang J and Zhang N: miR-203 suppresses bladder cancer cell growth and targets twist1. Oncol Res 26: 1155-1165, 2018.

22. Chi Y, Jin Q, Liu X, Xu L, He X, Shen Y, Zhou Q, Zhang J and Jin M: miR-203 inhibits cell proliferation, invasion, and migration of non-small-cell lung cancer by downregulating RGS17. Cancer Sci 108: 2366-2372, 2017.

23. Zierau O, Helle J, Schadyew S, Morgenroth Y, Bentler M Hennig A, Chittur S, Tenniswood M and Kretzschmar G: Role of miR-203 in estrogen receptor-mediated signaling in the rat uterus and endometrial carcinoma. J Cell Biochem 119: 5359-5372, 2018.

24. Du SL, Xu LY, Gao P, Liu QS, Lu FF, Mo ZH, Fan ZZ, Cheng XL and Dong ZH: miR-203 regulates DJ-1 expression and affects proliferation, apoptosis and DDP resistance of pancreatic cancer cells. Eur Rev Med Pharmacol Sci 23: 8833-8840, 2019

25. Lin XM, Chen H, Zhan XL: miR-203 regulates JAK-STAT pathway in affecting pancreatic cancer cells proliferation and apoptosis by targeting SOCS3. Eur Rev Med Pharmacol Sci 23: 6906-6913, 2019.

26. Greither T, Grochola LF, Udelnow A, Lautenschläger C, Würl P and Taubert H: Elevated expression of microRNAs 155, 203, 210 and 222 in pancreatic tumors is associated with poorer survival Int J Cancer 126: 73-80, 2010.

27. He S, Zhang G, Dong H, Ma M and Sun Q: miR-203 facilitates tumor growth and metastasis by targeting fibroblast growth factor 2 in breast cancer. Onco Targets Ther 9: 6203-6210, 2016.

28. Xu M, Gu M, Zhang K, Zhou J, Wang Z and Da J: miR-203 inhibition of renal cancer cell proliferation, migration and invasion by targeting of FGF2. Diagn Pathol 10: 24, 2015

29. Fu XF, Tian YZ and Dong XS: Effects of gemcitabine combined with heparanase inhibitor on invasion and migration of pancreatic cancer PANC-1 cells. Chin Med Clin v.15: 20-22, 2015.

30. Livak KJ and Schmittgen TD: Analysis of relative gene expression data using real-time quantitative PCR and the 2(-Delta Delta C(T)) method. Methods 25: 402-408, 2001.

31. Dąbowski K, Bogacka B, Tarnowski M and Starzyńska T: Pancreatic cancer microenvironment. Pol Merkur Lekarski 41: 296-302, 2016 (In Polish).

32. Coleman SJ, Chioni AM, Ghallab M, Anderson RK, Lemoine NR, Kocher HM and Grose RP: Nuclear translocation of FGFR1 and FGF2 in pancreatic stellate cells facilitates pancreatic cancer cell invasion. EMBO Mol Med 6: 467-481, 2014.
33. Mardhian DF, Vrynas A, Storm G, Bansal R and Prakash J: FGF2 engineered SPIONs attenuate tumor stroma and potentiate the effect of chemotherapy in 3D heterospheroidal model of pancreatic tumor. Nanotheranostics 4: 26-39, 2020.

34. Ren B, Cui M, Yang G, Wang H, Feng M, You L and Zhao Y: Tumor microenvironment participates in metastasis of pancreatic cancer. Mol Cancer 17: 108, 2018.

35. Fu XF, Dong XS, Gao F and Zhao HC: The expression of fibroblast grouth factor 2 in pancreatic cancer and its effect and mechanism on the invasion and metastasis of pancreatic cancer cells. J Shanxi Med Univ 87: 26-31, 2017.

36. Kostas M, Lampart A, Bober J, Wiedlocha A, Tomala J, Krowarsch D, Otlewski J and Zakrzewska M: Translocation of exogenous FGF1 and FGF2 protects the cell against apoptosis independently of receptor activation. J Mol Biol 430: 4087-4101, 2018.

37. Shirakihara T, Horiguchi K, Miyazawa K, Ehata S, Shibata T, Morita I, Miyazono K and Saitoh M: TGF- $\beta$ regulates isoform switching of FGF receptors and epithelial-mesenchymal transition. EMBO J 30: 783-795, 2011.

38. Chen X, Zhao H, Chen C, Li J, He J, Fu X and Zhao H: The HPA/SDC1 axis promotes invasion and metastasis of pancreatic cancer cells by activating EMT via FGF2 upregulation. Oncol Lett 19: 211-220, 2020.

39. Wang N, Zheng J, Chen Z, Liu Y, Dura B, Kwak M, Xavier-Ferrucio J, Lu YC, Zhang M, Roden C, et al: Single-cell microRNA-mRNA co-sequencing reveals non-genetic heterogeneity and mechanisms of microRNA regulation. Nat Commun 10: 95, 2019.

40. Tan S, Xia L, Yi P, Han Y, Tang L, Pan Q, Tian Y, Rao S, Oyang L, Liang J, et al: Exosomal miRNAs in tumor microenvironment. J Exp Clin Cancer Res 39: 67, 2020.

41. Parente P, Parcesepe P, Covelli C, Olivieri N, Remo A, Pancione M, Latiano TP, Graziano P, Maiello E and Giordano G: Crosstalk between the tumor microenvironment and immune system in pancreatic ductal adenocarcinoma: Potential targets for new therapeutic approaches. Gastroenterol Res Pract 2018: 7530619, 2018

42. Lee $M$ and Rhee I: Cytokine signaling in tumor progression. Immune Netw 17: 214-227, 2017.

43. Akl MR, Nagpal P, Ayoub NM, Tai B, Prabhu SA, Capac CM, Gliksman M, Goy A and Suh KS: Molecular and clinical significance of fibroblast growth factor 2 (FGF2 / bFGF) in malignancies of solid and hematological cancers for personalized therapies. Oncotarget 7: 44735-44762, 2016.

44. Shi K, Qiu X, Zheng W, Yan D and Peng W: miR-203 regulates keloid fibroblast proliferation, invasion, and extracellular matrix expression by targeting EGR1 and FGF2. Biomed Pharmacother 108: 1282-1288, 2018

45. Li B, Pi Z, Liu L, Zhang B, Huang X, Hu P, Chevet E, Yi P and Liu J: FGF-2 prevents cancer cells from ER stress-mediated apoptosis via enhancing proteasome-mediated Nck degradation. Biochem J 452: 139-145, 2013.

46. Turner $\mathrm{N}$ and Grose R: Fibroblast growth factor signalling: From development to cancer. Nat Rev Cancer 10: 116-129, 2010.

47. Mohammadi M, Olsen SK and Ibrahimi OA: Structural basis for fibroblast growth factor receptor activation. Cytokine Growth Factor Rev 16: 107-137, 2005.

48. Li J, Shan F, Xiong G, Chen X, Guan X, Wang JM, Wang WL, $\mathrm{Xu} \mathrm{X}$ and Bai Y: EGF-induced C/EBP $\beta$ participates in EMT by decreasing the expression of miR-203 in esophageal squamous cell carcinoma cells. J Cell Sci 127: 3735-3744, 2014.

49. Zhang K, Dai L, Zhang B, Xu X, Shi J, Fu L, Chen X, Li J and Bai Y: miR-203 is a direct transcriptional target of E2F1 and causes G1 arrest in esophageal cancer cells. J Cell Physiol 230: 903-910, 2015

This work is licensed under a Creative Commons Attribution-NonCommercial-NoDerivatives 4.0 International (CC BY-NC-ND 4.0) License. 